\title{
Circular RNA circNELL2 Acts as the Sponge of miR-127-5p to Promote Esophageal Squamous Cell Carcinoma Progression
}

This article was published in the following Dove Press journal: OncoTargets and Therapy

\section{Gang Xiong \\ Dingwei Diao \\ Di Lu \\ Xiguang Liu \\ Zhaoguo Liu \\ Shijie Mai \\ Siyang Feng \\ Xiaoying Dong \\ Kaican Cai}

Department of Thoracic Surgery, Southern Medical University Nanfang Hospital, Guangzhou City, Guangdong Province 510515, People's Republic of China
Correspondence: Kaican Cai Department of Thoracic Surgery, Southern Medical University Nanfang Hospital, No. 1838, Guangzhoudadao Road, Guangzhou City, Guangdong Province 510515, People's Republic of China

Email caican@smu.edu.cn
Introduction: Owing to its involvement in both the initiation and progression of various cancers, aberrant circular RNA (circRNA) expression has been researched extensively in the recent times. In the present study, we aim to investigate the effect of a novel circRNA has_circ_0025933 (circNELL2) in the progression of esophageal squamous cell carcinoma (ESCC).

Materials and Methods: Sanger sequencing and the detection of circNELL2 level after RNase R or actinomycin D treatment were performed to identify the existence of cirNELL2 in ESCC cells. WST, EDU staining and colony-formation assay were used to assess the proliferation while transwell assay was used to evaluate the migration of ESCC cells. Luciferase assay, RNA pull down and the FISH assay were performed to verify the interaction between circNELL2 and miR-127-5p as well as miR-127-5p and CDC6. Xenograft model was carried out to evaluate the effect of circNELL2 in vivo.

Results: circNELL2 was proved to exist in ESCC cells. The up-regulated expression of circNELL2 in the clinical ESCC specimens was also verified. Next, function studies suggested that circNELL2 knockdown inhibited the proliferation of ESCC cells in vitro and in vivo, while circNELL2 overexpression promotes that of ESCC cells. Besides, this study mechanically predicted and verified the target miR of circNELL2, which is miR-127-5p. It was found that miR-127-5p was capable of reversing the effect of circNELL2 on ESCC cells. Moreover, miR-127-5p was also found to target CDC6 to participate in the regulation of cell phenotype.

Discussion: circNELL2 promoted the progression of ESCC cells via sponging miR-127-5p, and it has the potential to serve as a novel prognostic and therapeutic target for ESCC.

Keywords: circular RNA circNELL2, miR-127-5p, esophageal squamous cell carcinoma

\section{Introduction}

Esophageal squamous cell carcinoma (ESCC) is one of the most common esophageal carcinoma accounts for more than $90 \%$ of it, with its incidence rising continuously in the recent years. Conventional therapeutic strategies of ESCC include surgery, hormone therapy and radioactive iodine therapy. ${ }^{1,2}$ Despite the advance in the development of these diagnosis and treatment strategies, the 5-year survival rate of patients with advanced ESCC is still under $60 \%{ }^{3}$ Thus, it is urgent to discover novel potential therapeutic targets which require us to have a much more comprehensive understanding of the molecular mechanism underlying ESCC progression.

Circular RNAs (circRNA) are a novel family of non-coding RNAs characterized by formation of closed loops without $5^{\prime}$ to $3^{\prime}$ polarity or polyadenylated tails 
covalently. ${ }^{4}$ They are highly conserved and abundant through different species for their stable structure which can resist the digestion of RNase $\mathrm{R}$ making them idea biomarkers for diagnosis and prognosis. ${ }^{5,6}$ Since early discovered, circRNAs were identified as splicing errors or by-product of incorrect RNA splicing with no value. However, with the deepening of research on them, they have been demonstrated to play crucial roles in the biological processes such as splicing regulation, ${ }^{7}$ sponging microRNA (miRNA), ${ }^{8,9}$ and regulating protein-RNA interactions. ${ }^{10}$ As in the field of cancer, circRNAs have been indicated to be dysregulated in various cancers including prostate cancer, ${ }^{11}$ colorectal cancer, ${ }^{12}$ hepatocellular carcinoma, ${ }^{13,14}$ as well as glioblastoma, ${ }^{15}$ and participate in the physiological processes of these cancers. Previous studies have introduced the function of hsa circ_0000654 and hsa_circ_0000337 in the progression of ESCC through diverse molecular mechanisms. ${ }^{16-18}$

Here, we identified circNELL2 (hsa_circ_0025933) which was spliced from exon 14 and exon 15 of the neural EGFL like 2 (NELL2) gene, and with the length of 294 nucleotides. In our study, we found the dysregulated expression of circNELL2 and its oncogenic role in ESCC through sponging miR-127-5p and further regulating the downstream CDC6 expression. These findings give us a better understanding of the function of circRNAs in ESCC progression and provide a novel potential therapeutic and diagnosis biomarker.

\section{Materials and Methods}

\section{Patient Specimens}

Twenty-five pairs of ESCC tissues and adjacent normal tissues were obtained from ESCC patients at the Nanfang Hospital. Upon collection, all samples were snap-frozen using liquid nitrogen and stored at a temperature of $-80^{\circ} \mathrm{C}$ until. All procedures were performed in accordance with the Declaration of Helsinki of the World Medical Association. The written informed consents have been provided by the patients. All experiments involving animals and human tissues were approved by the Ethics Committee of the Nanfang Hospital.

\section{Cell Culture}

ESCC cell lines (OE33 and FLO-1) were obtained from the ATCC (Manassas, VA, USA). All obtained cells were cultured in Dulbecco's Modified Eagle's Medium (DMEM) (Gibco, CA, USA) containing 10\% FBS (fetal bovine serum, Gibco, CA, USA) supplemented with $100 \mathrm{U} \cdot \mathrm{mL}^{-1}$ penicillin, and $100 \mathrm{mg} \cdot \mathrm{mL}^{-1}$ streptomycin (Invitrogen, CA, USA) in humid conditions with $5 \% \mathrm{CO}_{2}$ at a temperature of $37^{\circ} \mathrm{C}$.

\section{Plasmids Construction}

SiRNAs for circNELL2, the control, the miR-127-5p mimic, mimic control, miR-127-5p inhibitor, and inhibitor control are designed and synthesized by GenePharma (Shanghai, China). The shRNA against circNELL2 and negative control were also synthesized by GenePharma (Shanghai, China). In order to establish the overexpressing vectors of circNELL2 and CDC6, the coding sequence of human circNELL2 and CDC6 were synthesized and inserted into the pLCDH-ciR and pcDNA3 vector, respectively (Bioco Biotech Co, Tianjin, China). Furthermore, wild type and mutant sequence that contains the binding site of miR-127-5p from circNELL2 and CDC6 were synthesized and cloned into pGL3 luciferase reporter vectors (Promega, CA, USA).

\section{Oligonucleotides Transfection and Stable Transfection}

Lipofectamine 3000 (Invitrogen, CA, USA) was used to transfect Oligonucleotides at a concentration of $60 \mathrm{nmol} / \mathrm{L}$, the procedure was done in accordance with the manufacturer's instructions. In order to establish stable cell lines, all the cells subjected to transfection were subsequently selected with $2 \mu \mathrm{g} / \mathrm{mL}$ puromycin continuously for a period of 2 weeks.

\section{WST Assay}

WST assay was performed to evaluate the cell viability, the procedure followed instructions from the manufacturer. The cells were seeded in 96 -well plates $\left(5 \times 10^{3} /\right.$ well). Following the treatment, cell viability was determined every 24 hours over a period of 3 days. Using a Microplate Reader (Bio-Rad, CA, USA), absorbance was detected at $490 \mathrm{~nm}$. Each assay was performed at least three times.

\section{Colony-Formation Assay}

The cells were treated with indicated conditions, and then seeded in 12-well plates (100/well). Following an incubation period of 2 weeks, crystal violet $(0.05 \%$, Beyotime, Shanghai, China) was used to stain the colonies. Colonies containing more than 50 cells were counted. 


\section{Transwell Assay}

Transwell assay was used to detect cell invasion ability (Millipore, MA, USA). Cells were treated with indicated conditions, and then the treated cells were seeded on the upper insert coated with 2\% Matrigel (BD Biosciences, NY, USA) in 24-well plates. The upper insert was filled with medium lacking serum, and the lower chamber was filled with $600 \mu \mathrm{L}$ of DMEM supplemented with $10 \%$ FBS. Following a period of $24 \mathrm{hrs}$ of incubation, cells invaded to the lower chambers were fixed with methanol, stained with crystal violet.

\section{Luciferase Activity Assay}

The wild-type or mutant seed sequence at the predicted region of circNELL2 and CDC6 were synthesized and cloned into the pGL3 Luciferase Reporter vectors (Promega, CA, USA) at the KpnI and BamHI sites. Following this, the ESCC cells were co-transfected with miR-127-5p mimics, or mimic control, together with pGL3 vectors, which contained the WT or mut binding region of circNELL2 and CDC6. In addition, TRL-SV40 plasmid (Promega, CA, USA) was also transfected as a normalizing control. Dual-Luciferase Assay was used to harvest the cells for detecting the activity of luciferase (Promega, WI, USA) at $48 \mathrm{hrs}$ following the transfection.

\section{Quantitative Real-Time PCR}

The miRNeasy Mini Kit (Qiagen, Germany) was used to extract mRNA and miRNA from the tissues and cells. cDNA was attained by the means of reverse transcription assay with the use of the reverse transcriptase, and Oligod (T) was employed as the primer. With regards to the cDNA synthesis of miR-127-5p, a particular reverse transcription primer was used. QRT-PCR was carried out using a SYBR-Green Supermix kit in a biorad IQ5 system (CA, USA). U6 small nuclear RNA (U6-snRNA) and GAPDH were used as the internal control for the purpose of evaluating the corresponding relative expression levels of miR-127-5p, circNELL2, and mRNAs.

\section{Western Blotting}

RIPA buffer (Jiancheng, Nanjing, China) was used to extract the proteins of tissue samples and cells. The protein extracts were separated on a sodium dodecyl sulfatepolyacrylamide gels (SDS-PAGE), which were further transferred to PVDF membranes. Next, the blots were incubated in 5\% nonfat dry milk in phosphate-buffered saline (PBS) with $0.1 \%$ Tween 20 for a duration of $2 \mathrm{hrs}$ followed by incubating with primary antibodies (Proteintech, Wuhan, China) overnight at a temperature of $4^{\circ} \mathrm{C}$. Next, the blots were incubated in horseradish peroxidase conjugated secondary antibody (Cell Signaling Technology, USA) for a duration of $2 \mathrm{hrs}$. Following this, the proteins were visualized using an ECL kit (Jiancheng, Nanjing, China), and quantified using the Image $\mathrm{J}$ software.

\section{Fluorescence in situ Hybridization (FISH)} Alexa Fluor 555-labeled circNELL2 probes, and Alexa Fluor 488-labeled miR-127-5p probes were designed and synthesized by RiboBio (Guangzhou, China). A fluorescent in situ Hybridization Kit (RiboBio, Guangzhou, China) was used to carry out the FISH experiment. A total of $1 \times 10^{5}$ cells were seeded onto autoclaved glass slides and cultured for a period of 24 hours. Following a fixing procedure with 4\% paraformaldehyde for a duration of 20 minutes, was followed by permeabilization with $0.5 \%$ Triton X-100 for a duration of 10 minutes, the cells were then cultured overnight at a temperature of $37^{\circ} \mathrm{C}$. Finally, the slides were incubated with DAPI to stain cell nuclear before being observed under a fluorescence microscope (Leica, Wetzlar, Germany).

\section{RNA Pull Down}

Biotin-labeled miR-127-5p probe and control probe were synthesized by Sangon Biotech (Shanghai, China). Probecoated beads were generated by co-incubating the probe with streptavidin-coated beads (Invitrogen, CA, USA) at a temperature of $25^{\circ} \mathrm{C}$ for 2 hours. ESCC cells were collected and lysed and incubated with miR-127-5p probes overnight at $4^{\circ} \mathrm{C}$. Following this, the beads were eluted and the complex was purified with TRIzol (Takara, Dalian, China). Then the abundance of circNELL2 and CDC6 was analyzed using qRT-PCR.

\section{Immunohistochemistry (IHC)}

The tumor tissues were fixed in 4\% paraformaldehyde for $24 \mathrm{~h}$; next, they were dehydrated in a graded alcohol series and embedded in paraffin. Following this, they were cut into $5 \mu \mathrm{m}$ sections. The sections were deparaffinized, rehydrated with a graded alcohol series, and then incubated in $96^{\circ} \mathrm{C} 0.01 \mathrm{~mol} / \mathrm{L}$ sodium citrate buffer to allow the retrieval of antigen. Following incubation, $5 \% \quad \mathrm{H}_{2} \mathrm{O}_{2}$ was applied for quenching endogenous peroxidase activity. In order to reduce non-specific binding, the sections were blocked with $10 \%$ non-immune goat serum. The sections were incubated with primary antibodies overnight at $4^{\circ} \mathrm{C}$. By following the manufacturer's instructions (Beyotime, 
Shanghai, China), immunostaining was performed using streptavidin-peroxidase and diaminobenzidine (DAB). The sections were mounted with gummi after staining the nucleus with hematoxylin, after being dehydrated in a graded alcohol series and pellucidum in xylene.

\section{Xenograft Model}

As mentioned above, stably expressing or knocking down circNELL2 cell lines OE-33 and FLO-1 were established. Cells were harvested and resuspended in DMEM medium. The nude mice were injected at the posterior flank with a total of $3 \times 10^{6} / 100 \mu \mathrm{L}$ cell subcutaneously. Every 3 days, the tumor size was monitored by measuring the length $(\mathrm{L})$ and width (W) with calipers. After 28 days, the tumors were excised from the sacrificed mice and weighed. All experiments involving animals were approved by the Ethics Committee of the Nanfang Hospital and followed by Guidelines for the ethical review of laboratory animal welfare People's Republic of China National Standard GB/T 35892-2018.

Statistical analysis

All data are presented as the means \pm standard deviation (SD). The statistical analyses were performed using SPSS 20 software (Abbott Laboratories, Chicago, IL, USA). Data were analyzed with one-way ANOVA and Student's $t$ test. $\mathrm{P}<0.05$ was considered to be statistically significant. Linear correlation analyses were performed to determine correlations between circNELL2, miR-127-5p and CDC6 expression levels. All experiments were performed independently triplicates.

\section{Results}

\section{Identification of circNELL2 in ESCC}

The 294-bp circNELL2 was looped by circularization of exon 14 and 15 of the NELL2 gene, which was located at chromosome $12 \mathrm{p} 22$ referring to the sequence from circBase. In the present study, it was further verified by the head-to-tail splicing through sanger sequencing (Figure 1A). It is known that circRNAs are stable for their ring structure. In order to evaluate the stability of circNELL2, the present study evaluated the amount of circNELL2 of OE-33 and FLO-1 cells under RNase $\mathrm{R}$ treatment. As indicated in Figure 1B, the amount of linear mRNA of NELL2 was significantly decreased under the RNase R treatment. However, there was no evidence of alteration of circNELL2 under digestion of RNase R. Furthermore, we assessed the levels of linear NELL2 and circNELL2 under the treatment of Actinomycin D. The results revealed that treatment with actinomycin D had no significant effect on the level of circNELL2 at different times; however, the level of linear NELL2 was significantly decreased after actinomycin D (Figure 1C). Furthermore, cDNA and gDNA were extracted separately from OE-33 and FLO-1 cells, and they were subjected to nucleic acid electrophoresis detection. According to the results, circNELL2 could be detected in cDNA; however, they were undetectable in gDNA (Figure 1D). Once it was identified that circNELL2 existed, the next step involved evaluating the expression level of circNELL2 in ESCC. It was significantly up-regulated in the ESCC tissues compared to that in normal tissues (Figure 1E).

\section{Knock Down of circNELL2 Inhibited the Proliferation, Colony Formation, and the Migration in ESCC Cells}

Following this, the study carried out loss of function studies including cell proliferation, colony formation, and cell migration. In order to further detect biological functions of circNELL2, two siRNAs targeting the back-splice sequence of circNELL2 was designed to knock down the expression of circNELL2. Firstly, the level of circNELL2 was detected to confirm whether the two siRNAs were capable of inhibiting the expression of circNELL2. According to the results, both siRNAs successfully decreased the expression level of circSMAD4A (Figure 2A). Subsequently, CCK-8 and EDU staining were performed to evaluate the proliferation of OE-33 and FLO-1 cells. A notable inhibition of OE-33 and FLO-1 cell proliferation subjected to circNELL2 knock down was observed (Figure 2B and C). In addition, colony formation and transwell assay indicated that circNELL2 knock down significantly reduced colony-formation ability and cell migration of ESCC cells (Figure 2D and E).

\section{circNELL2 Works as an Efficient miR-127-5p Sponge in ESCC}

Generally, it has been established that circRNAs are capable of sponging miRNAs and subsequently abrogate the function of the corresponding miRNAs. On this basis, it was decided to explore whether circNELL2 sponge miRNAs. Circinteractom databases were used to predict the miRNA targets of circNELL2, among which, miR-127-5p was selected as a potential target. Figure $3 \mathrm{~A}$ shows the target region between circNELL2 and miR-127-5p. Luciferase 


\section{A}
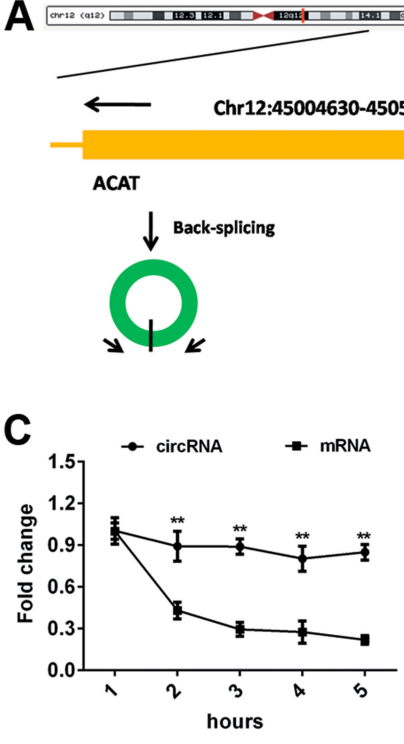

B
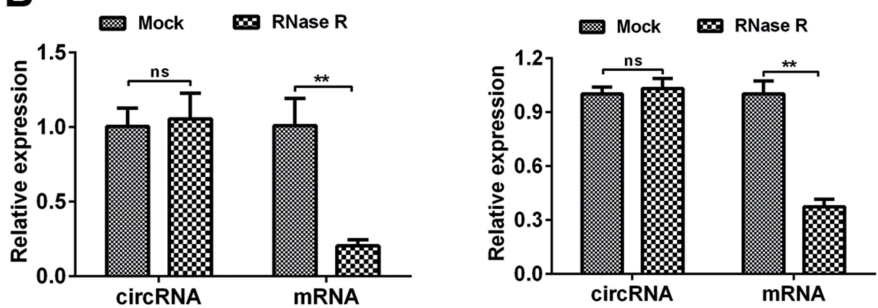

D Marker CDNA gDNA CDNA gDNA

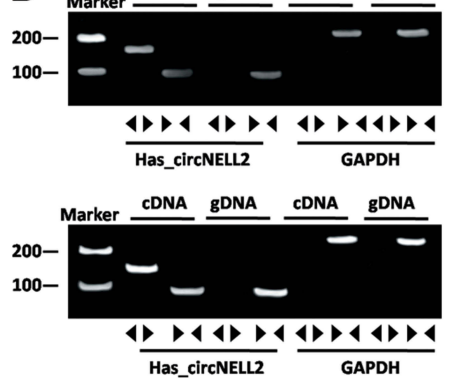

E

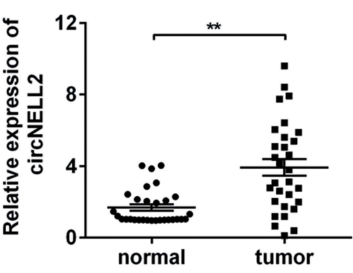

Figure I The identification of circNELL2. (A) circNELL2 was back-spliced by exon 14 and exon 15 of the neural EGFL like 2 (NELL2) gene with the length of 294 nucleotides. The existence of circNELL2 were confirmed by sanger sequencing, the arrow shows the head-to-tail splicing junction site of circNELL2. (B) After RNase $R$ treatment, the expression of linear RPLI3 mRNA and circNELL2 were detected using qPCR. (C) qRT-PCR analysis of RPLI 3 mRNA and circNELL2 in cardiomyocytes after treatment with Actinomycin D for I, 2, 3, 4, 5 h, respectively. (D) The existence of circNELL2 was validated by RT-PCR. Divergent primers amplified circNELL2 in cDNA but not genomic DNA (gDNA). (E) qPCR was performed to evaluate the expression of circNELL2 in ESCC tissues. $* \mathrm{P}<0.05$, $* * P<0.01$.

A

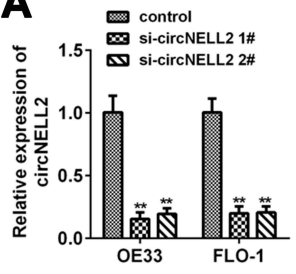

B
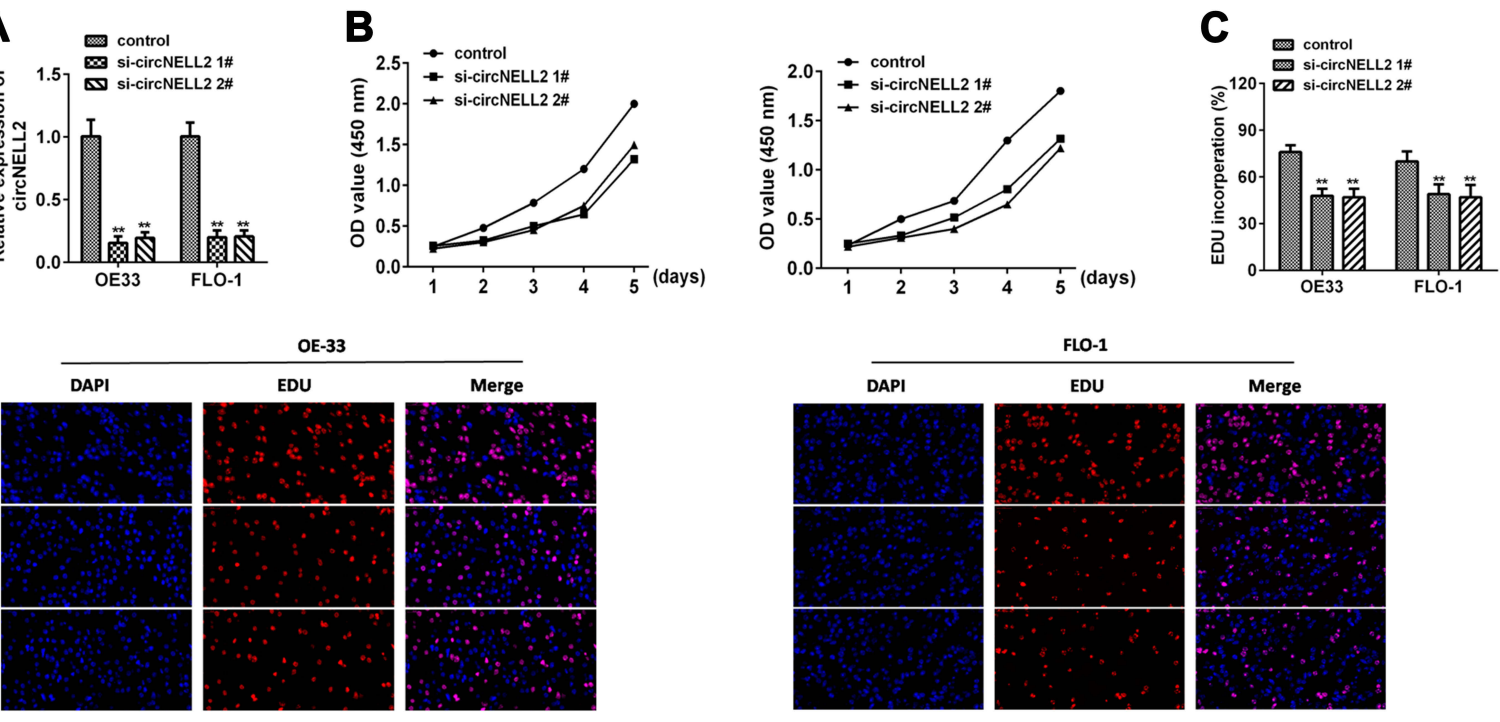

OE-33
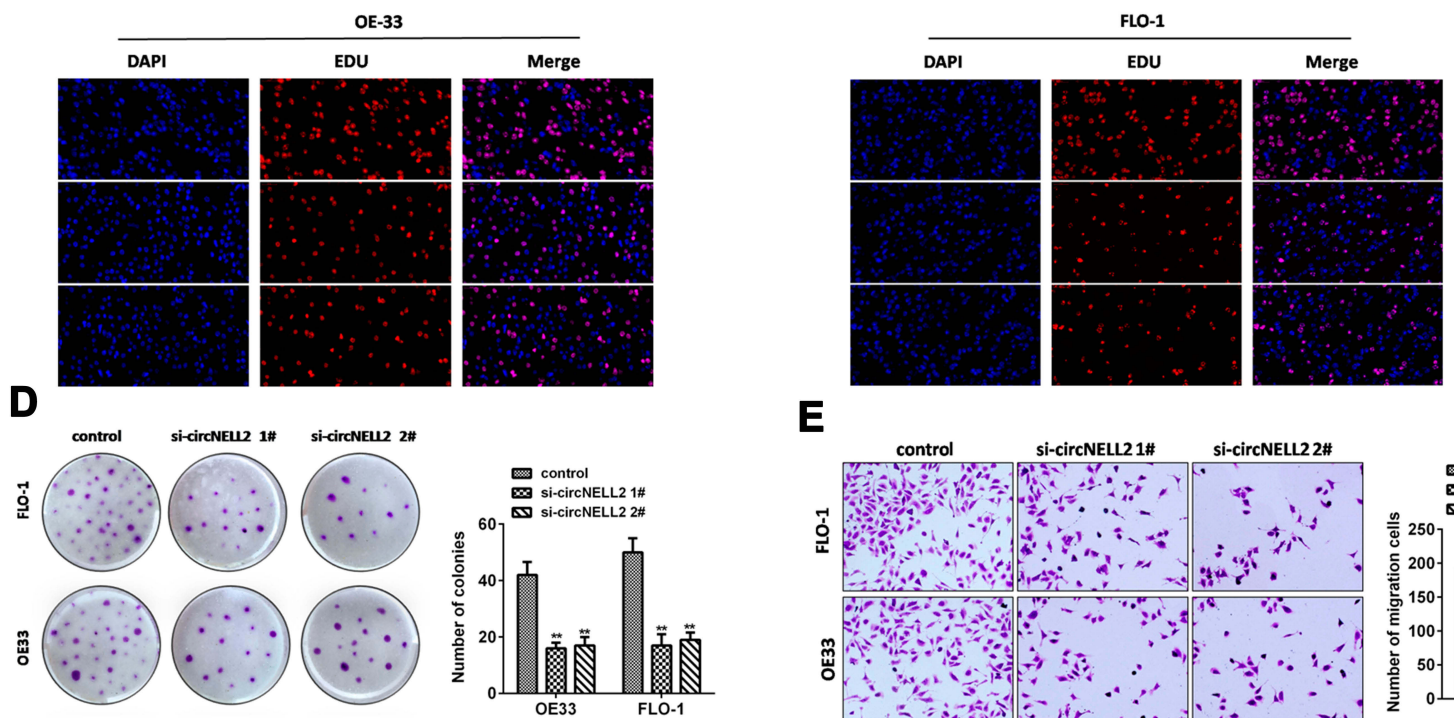

E

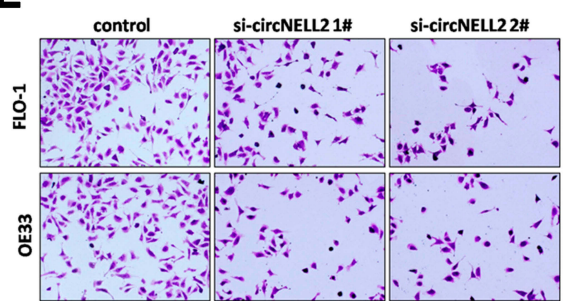

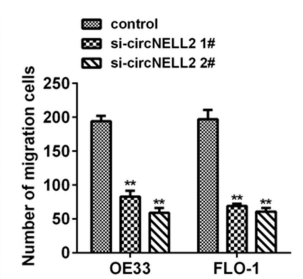

Figure 2 circNELL2 knock down attenuates cell proliferation, colony formation, migration and invasion of ESCC cell lines. (A) qPCR was performed to evaluate the expression level of circNELL2 in ESCC cell under different treatment. Cell viability of OE33 and FLO-I cells was investigated using (B) EDU staining and (C) WST assay. (D) Clonability of OE33 and FLO-I cells was investigated by colony-formation assay. (E) Migration ability of OE33 and FLO-I cells were detected by transwell experiment. $* \mathrm{P}<0.05$. 


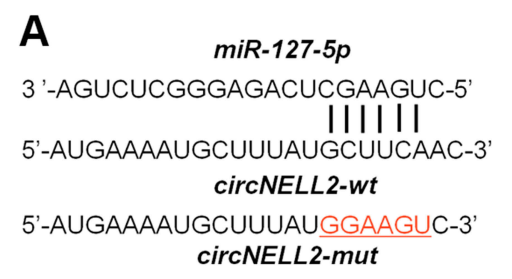

D

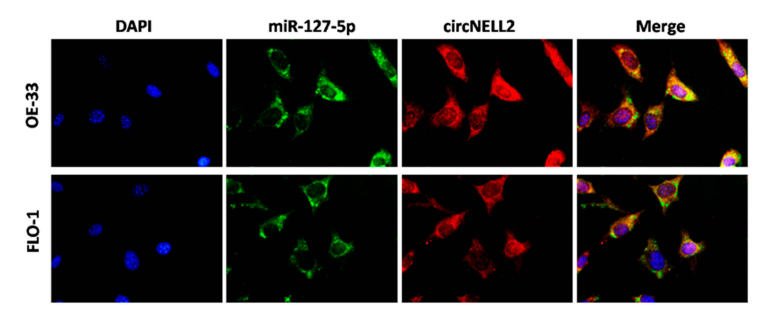

\section{B}

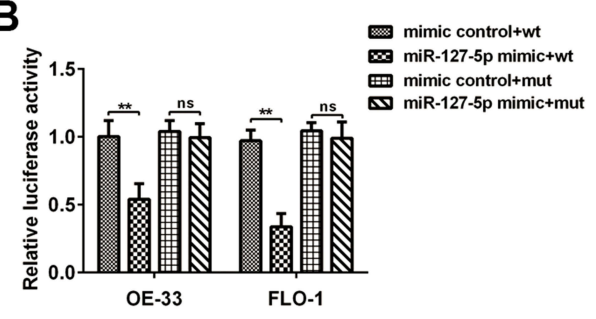

E

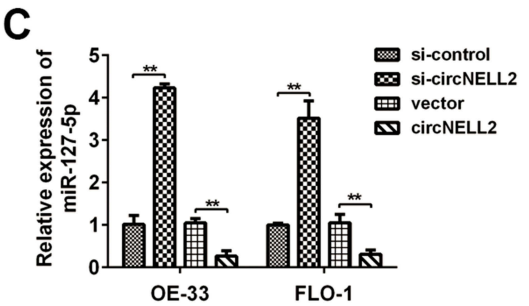

$\mathbf{F}$

Figure 3 circNELL2 directly targets miR-127-5p. (A) circNELL2 was predicated as a potential target gene of miR-I27-5p, wild and mutant type of targeting seed sequences between circNELL2 and miR-127-5p are shown. (B)Luciferase activities were used to investigate the targeting of circNELL2 and miR-127-5p. (C) qPCR was performed to evaluate the expression of miR-I27-5p in response to circNELL2 overexpression or knock down. (D) FISH experiment using the probe of miR-I27-5p and circNELL2 were carried out to evaluate the co-location of them. (E) RNA pull down using the specific probe of miR-127-5p was performed to evaluate the interaction between miR-485-3p and circNELL2. (F) Pearson analysis was performed to evaluate the correlation between miR-127-5p and circNELL2. *P $<0.05$.

activity assay in both OE-33 and FLO-1 cells was carried out. The findings indicate that miR-127-5p evidently downregulated the luciferase activity co-transfected with pGL33'UTR of circNELL2; however, it does not include the pGL3-3'UTR-mut (Figure 3B). qPCR It was further confirmed through analyses that circNELL2 overexpression decreased the level of miR-127-5p, whilst the knock down of circNELL2 increased that of miR-127-5p (Figure 3C). Moreover, the FISH experiment was performed to detect the location of circNELL2 and miR-127-5p. As illustrated in Figure 3D, co-location of circNELL2 and miR-127-5p in cytoplasm was observed. In order to further detect whether circNELL2 could bind directly to miR-127-5p endogenously, RNA pull down was carried. It was found that biotinlabeled miR-127-5p enriched circNELL2 which indicated the binding between circ NELL2 and miR-127-5p (Figure 3E). Pearson analysis indicated a negative correlation between circNELL2 and miR-127-5p (Figure 3F).

\section{miR-I 27-5p Reversed the Function of circNELL2 in ESCC}

With the aim of investigating the role of miR-127-5p in ESCC, and the relation between miR-127-5p and circNELL2, a rescue experiment was carried out. OE-33 and FLO-1 cells were transfected with circNELL2, miR-127-5p or with both. Firstly, the expression level of miR-127-5p under different kinds of oligonucleotide transfection was evaluated. It was observed that circNELL2 overexpressing vector remarkably decreased the level of miR-127-5p whilst miR-127-5p overexpressing vector increased the level of that. It was also found that the co-transfection of circNELL2 and miR-127-5p reversed the down-regulation of miR-127-5p which was induced by circNELL2 overexpression (Figure 4A). Function studies indicated that circNELL2 had the capability of promoting cell proliferation, colony formation, and migration of ESCC cells. Contrarily, miR-127-5p overexpression inhibited cell proliferation, colony formation, and migration of ESCC cells. Finally, co-transfection of circNELL2 and miR-127-5p reversed the effect of circNELL2 on cell proliferation, colony formation, and migration of ESCC cells (Figure 4B-H).

\section{miR-127-5p Directly Targets CDC6 in ESCC}

In order to predict the targets of miR-127-5p, Miranda and TargetScan databases were used. Among these, CDC6 was selected as a potential target due to its existence in both databases (Figure 5A). Figure 5B shows the target region between miR-127-5p and CDC6. Luciferase activity assay was carried out in both OE-33 and FLO- 1 cells. As indicated by the findings, miR-127-5p evidently down-regulated the luciferase activity co-transfected with pGL3-3'UTR of CDC6; however, the pGL3-3'UTR-mut was not found to be so (Figure 5C). The qPCR and Western blot analyses further confirmed that miR-127-5p overexpression decreased the 
A

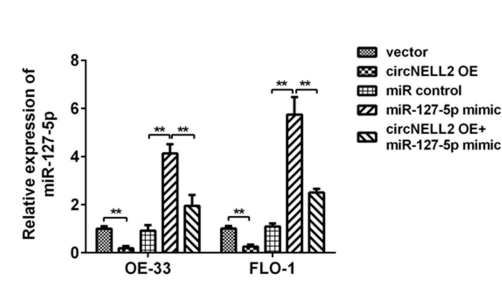

D
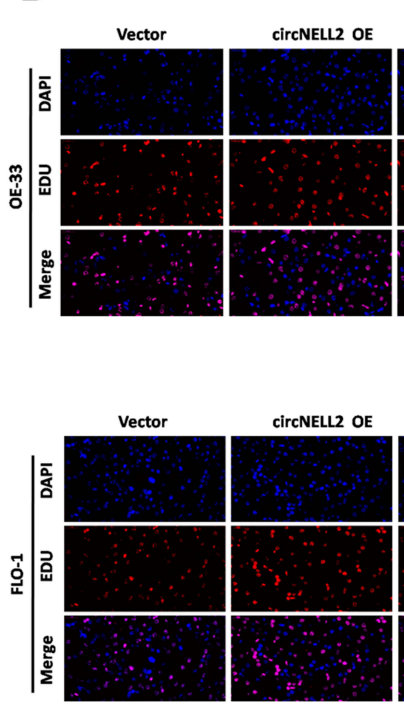

B

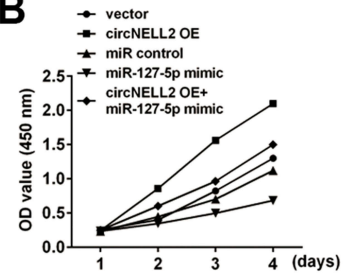

circNELL2 OE

miR control
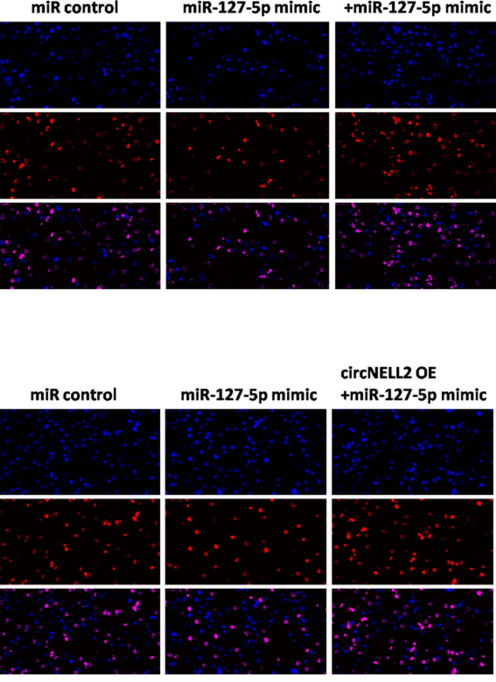

circNELL2 OE tmiR-127-5p mimic

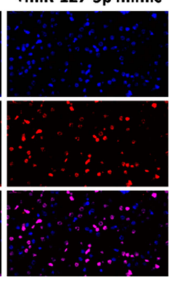

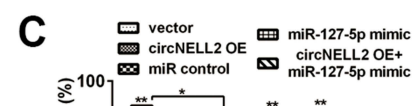

产

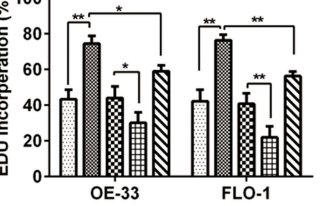

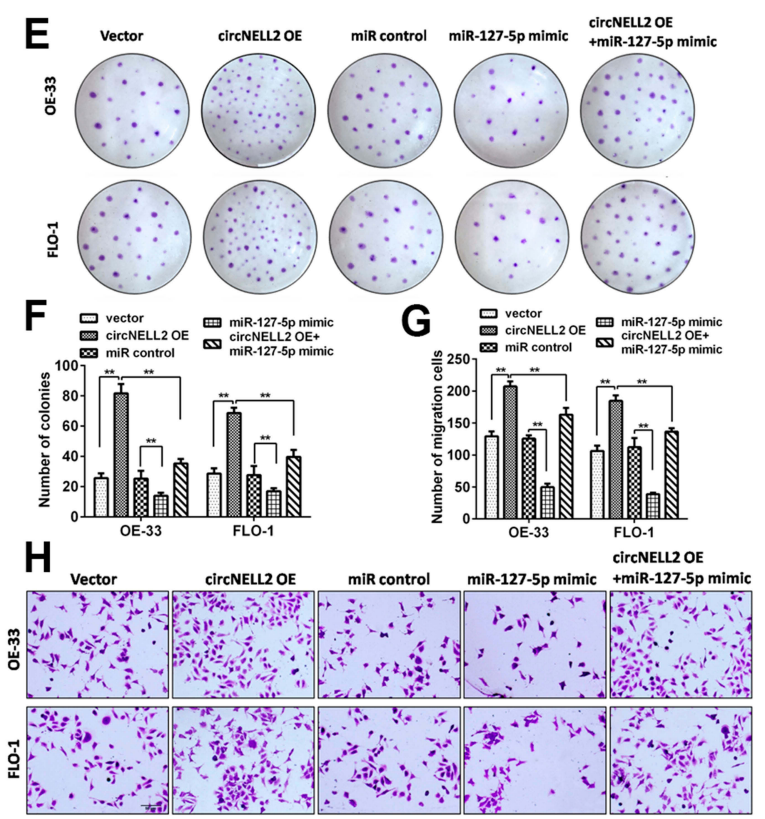

Figure 4 Restoration of miR-I27-5p and knock down of RELA significantly rescues circNELL2-mediated regulation of cellular effects. (A) qPCR was performed to evaluate the expression of RELA under different treatment. OE33 and FLO-I cell viability was detected using (B) WST assay and (C, D) EDU staining assay. (E, F) Clonability of OE33 and FLO-I cells was investigated by colony-formation assay. (G, H) Migration ability of OE33 and FLO-I cells were detected by transwell experiment. *P < 0.05 .

level of CDC6, it was also revealed that knock down of miR127-5p increased the level of CDC6 (Figure 5D, E). In order to further detect whether miR-127-5p could directly bind to CDC6 endogenously, RNA pull down was carried again. It was also found that biotin-labeled miR-127-5p enriched CDC6 which indicated the binding between CDC6 and miR-127-5p (Figure 5F). qPCR results revealed that circNELL2 silencing down-regulated the expression of CDC6 (Figure 5G). Pearson analysis showed a negative correlation between miR-127-5p and CDC6, and a positive correlation between circNELL2 and CDC6 (Figure 5H and I).

\section{CDC6 Was Up-Regulated in ESCC}

In order to further explore the potential effect of CDC6 in ESCC, the expression level of CDC6 in ESCC tissues was evaluated. Firstly, qPCR analysis was carried out, and it was found that CDC6 was significantly up-regulated in ESCC tissues compared to that in normal tissues (Figure 6A) and cell lines (Figure 6B). Additionally, pan-cancer analysis was performed using the data from the StarBase dataset, the analysis showed a notable elevation of CDC6 level in ESCC specimens (Figure 6C). Further confirmation of the upregulated expression of CDC6 in ESCC was provided by IHC analysis (Figure 6D).

\section{circNELL2 Affects ESCC Growth and Proliferation in vivo}

In order to explain the role of circNELL2 in the regulation of ESCC, the xenograft nude mice model was developed by means of subcutaneously injecting genetically modified OE-33 and FLO-1 cells. When compared with the vector and si-nc group, the circNELL2 overexpressing vector transfection substantially promoted the tumor growth of ESCC, this is evident from both the tumor growth curve and the weight of the tumor (Figure $7 \mathrm{~A}-\mathrm{C}$ ).

\section{Discussion}

Circular RNAs are a novel class of non-coding RNAs, they have not been extensively studied so far in ESCC. In the present study, a novel circular RNA named 
A

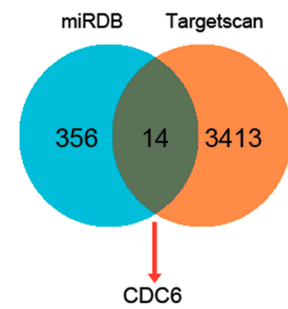

D

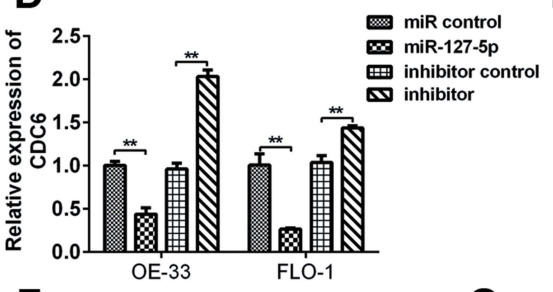

$\mathbf{F}$

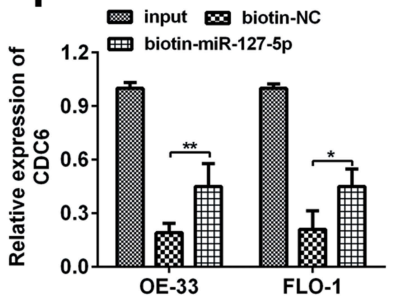

B

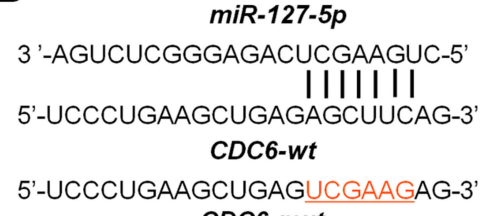

CDC6-mut
C

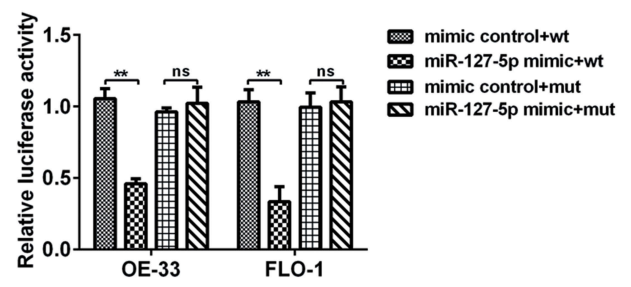

$E$

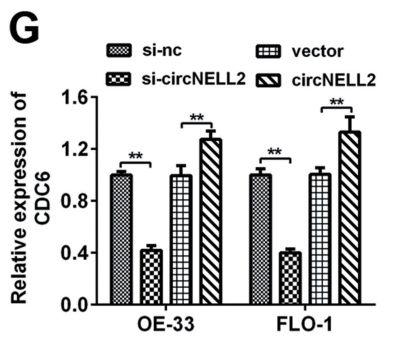

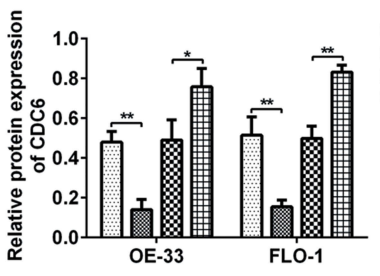

$\mathrm{H}$

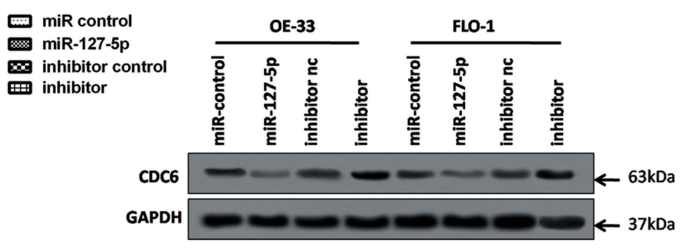

I

Figure 5 miR-127-5p directly targets CDC6 in ESCC cells. (A) The potential target gens were predicted by Targetscan, MiRDB. (B) CDC6 was predicated as a potential target gene of miR-127-5p, wild and mutant type of targeting seed sequences between CDC6 and miR-127-5p are shown. (C) Luciferase activities were used to investigate the targeting of CDC6 and miR-127-5p. (D, E) qPCR and Western blot assays were performed to evaluate the mRNA and protein expression of CDC6 in response to miR127-5p overexpression or knock down. (F) RNA pull down was carried out to detect the binding of miR-127-5p and CDC6. (G) qPCR was used to assess the expression of CDC6. $(\mathbf{H}, \mathbf{I})$ Pearson analysis was performed to evaluate the correlation between miR-127-5p and CDC6 as well as CDC6 and circNELL2. *P < 0.05 vs miR control, mimic control+wt and biotin-nc.

circNELL2 base was identified. It was known to upregulate in human ESCC tissues and cell lines. ${ }^{19}$ Functionally, this study demonstrated that knockdown of circNELL2 has the potential to inhibit cell proliferation as well as migration in vitro and in vivo, contrastingly, the up-regulation of circNELL2 exerted the opposite effects. Mechanistically, circNELL2 could function as a ceRNA through harboring miR-127-5p to abolish the suppressive effect on the target gene CDC6 in ESCC progression.

Circular RNAs participate in the regulation of physiological or physiological processes of cancer cells through its main mechanism of acting as a sponge for microRNAs. ${ }^{8}$ In the previous studies, circRNAs such as hsa_circ_0000654, hsa_circ_0000337, and circ-PRKCI have been reported to possess biological functionalities in ESCC by binding to miR-149-5p, miR-670-5p, and miR-3680-3p correspondingly. It was first identified that the role of miR-127-5p in ESCC was a target miRNA of circNELL2.

There are relatively few studies on miR-127-5p. It can be sponged by other circRNAs such as circ_0136474, ${ }^{20}$ circRNA.33186, ${ }^{21}$ and CircUSP45. ${ }^{22}$ The research of
miR-127-5p is mainly in the field of osteology. MiR-127$5 \mathrm{p}$ contributes to the pathogenesis of Osteoarthritis via targeting circRNA.33186 and CircUSP45. ${ }^{21-23}$ In addition, it regulates osteopontin (OPN)-mediated proliferation of human chondrocytes through PI3K/Akt pathway. ${ }^{24}$ For example, miR-127 was found to be downregulated in breast cancer. ${ }^{25}$ MicroRNA-127-5p targets the biliverdin reductase $\mathrm{B} /$ nuclear factor- $\mathrm{kB}$ pathway to suppress cell growth in hepatocellular carcinoma cells. ${ }^{26}$

In the present study, it was firstly found that miR-127-5p was down-regulated in ESCC tissues and cell lines, it was also found to be negatively correlated with circNELL2. Gain of function study showed an anti-cancer effect of miR-127-5p in ESCC which has not been elucidated before. In order to further investigate the effect of miR-127-5p in ESCC, a loss of function and in vivo study should be carried out following this research. In addition, CDC6 was predicted and verified as the direct target of miR-127-5p.

Cell division cycle $6(\mathrm{Cdc} 6)$ is a vital mediator of DNA replication, it plays a crucial role in the activation and maintenance of the cell cycle. ${ }^{27}$ The best-characterized 
A

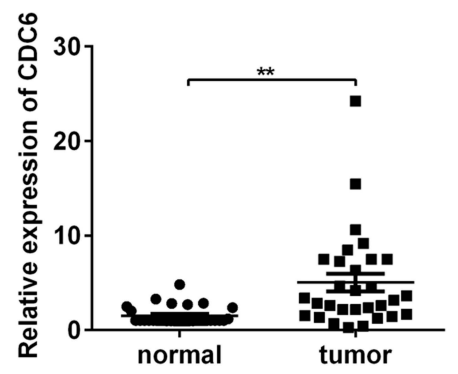

C

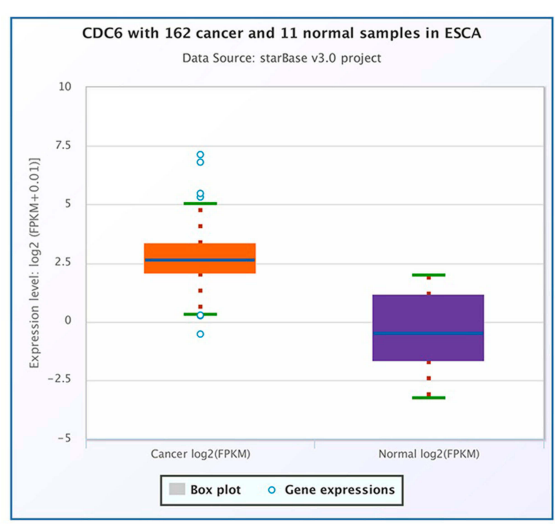

B
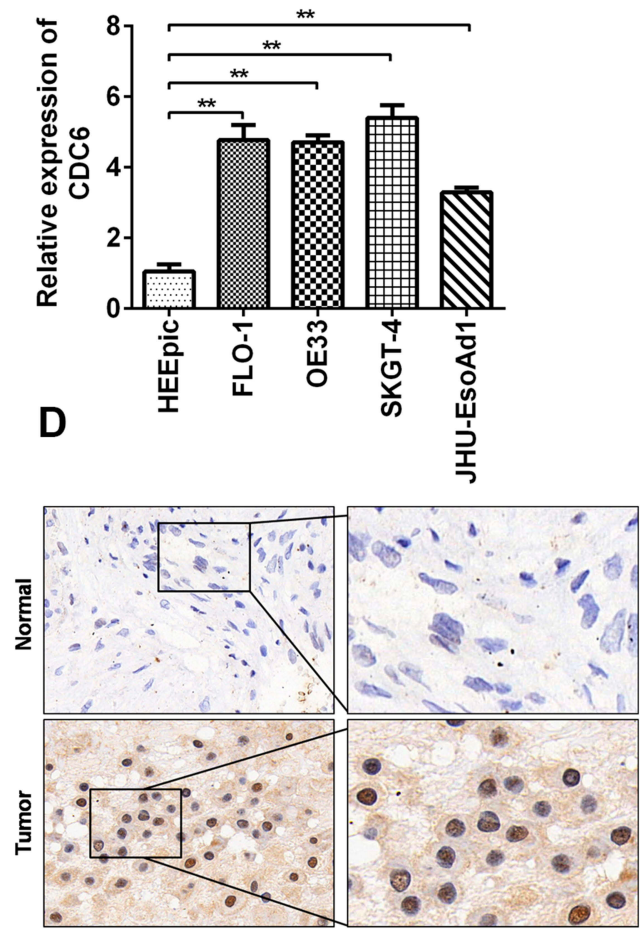

Figure 6 CDC6 is up-regulated in ESCC. qRT-PCR analysis of CDC6 in (A) ESCC tissues and (B) cell lines were assessed. (C) Analysis based on the data from starbase dataset was performed to show that $C D C 6$ was up-regulated in ESCC tissues. (D) $I H C$ was further carried out to confirmed the expression of CDC6 in tumor tissues. $* \mathrm{P}<0.05, * * \mathrm{P}<0.01$.

A

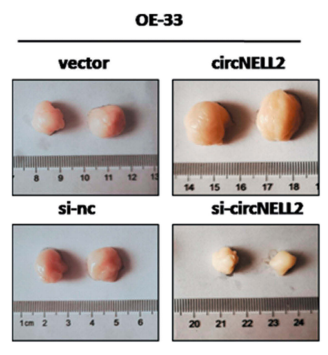

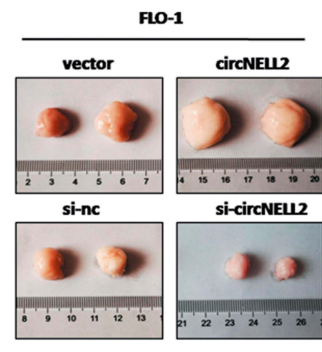

C

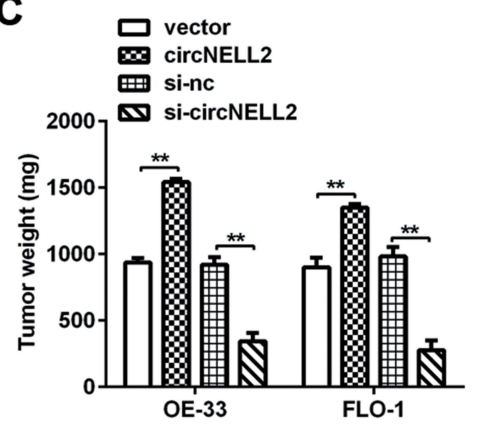

B

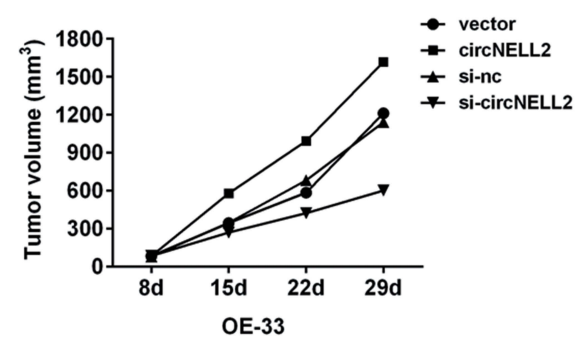

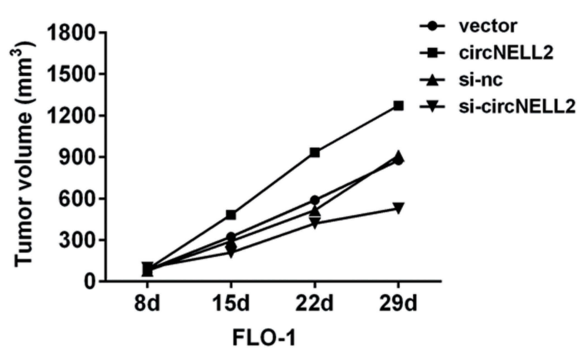

Figure 7 circNELL2 promote xenograft tumour growth in vivo. A xenograft tumour model was established by subcutaneously injecting OE33 and FLO-I cells stably overexpressing or knocking down circNELL2 along with their blank control into the dorsal flank of nude mice. (A) Tumour image was shown in each group. (B) Tumour weights was weighed. (C) The growth curves of the tumors in each group was made. ${ }^{*} \mathrm{P}<0.05$. 
function of Cdc6 is the assembly of pre-replicative complexes (pre-RC) at origins to initiate DNA replication in the G1 phase. ${ }^{28-30}$ Even though Cdc6 is widely known to be correlated with the progression of human cancers, ${ }^{8,17-19}$ the exact effect and mechanism of Cdc6 on the development of cancer is yet to be elucidated. Particularly, the role of CDC6 has not been studied before.

It was demonstrated that CDC6 was significantly upregulated due to the $\mathrm{qPCR}$ and $\mathrm{IHC}$ results in addition to the data analysis based on Star Base. The present study speculates that CDC6 exerts a critical role in the development of ESCC. However, further functional studies should be carried out to conclusively prove it. Furthermore, it was also confirmed that miR-127-5p targets CDC6 in ESCC which is a unique outcome of this study. Rescue experiments should also be performed to verify this relationship.

In summary, the current investigation shed light on the critical role of circNELL2 and miR-127-5p in the progression of ESCC by the means of regulating cell proliferation, colony formation, and migration. There have not been any prior studies that have thoroughly investigated the biological functions of circNELL2, and the underlying mechanism in ESCC as performed in this study. The findings in this study are likely to provide potential novel targets such as circNELL2 or miR-127-5p for effective therapies against esophageal squamous cell carcinoma.

\section{Disclosure}

The authors report no conflicts of interest in this work.

\section{References}

1. Agrawal N, Akbani R, Aksoy B. Integrated genomic characterization of papillary thyroid carcinoma. Cell. 2014;159(3):676-690. doi:10.1016/j.cell.2014.09.050

2. Xing M. Molecular pathogenesis and mechanisms of thyroid cancer. Nat Rev Cancer. 2013;13(3):184-199. doi:10.1038/nrc3431

3. Wu DM, Wang S, Wen X, et al. LncRNA SNHG15 acts as a ceRNA to regulate YAP1-Hippo signaling pathway by sponging miR-200a-3p in papillary thyroid carcinoma. Cell Death Dis. 2018;9(10):947. doi:10.1038/s41419-018-0975-1

4. Jeck WR, Sharpless NE. Detecting and characterizing circular RNAs. Nat Biotechnol. 2014;32(5):453-461. doi:10.1038/nbt.2890

5. Qu S, Zhong Y, Shang R, et al. The emerging landscape of circular RNA in life processes. RNA Biol. 2017;14(8):992-999. doi:10.1080/ 15476286.2016.1220473

6. Qu S, Yang X, Li X, et al. Circular RNA: a new star of noncoding RNAs. Cancer Lett. 2015;365(2):141-148. doi:10.1016/j. canlet.2015.06.003

7. Ashwal-Fluss R, Meyer M, Pamudurti NR, et al. circRNA biogenesis competes with pre-mRNA splicing. Mol Cell. 2014;56(1):55-66. doi:10.1016/j.molcel.2014.08.019
8. Hansen TB, Jensen TI, Clausen BH, et al. Natural RNA circles function as efficient microRNA sponges. Nature. 2013;495 (7441):384-388. doi:10.1038/nature11993

9. Du WW, Yang W, Liu E, Yang Z, Dhaliwal P, Yang BB. Foxo3 circular RNA retards cell cycle progression via forming ternary complexes with p21 and CDK2. Nucleic Acids Res. 2016;44 (6):2846-2858. doi:10.1093/nar/gkw027

10. Li Z, Huang C, Bao C, et al. Exon-intron circular RNAs regulate transcription in the nucleus. Nat Struct Mol Biol. 2015;22 (3):256-264. doi:10.1038/nsmb.2959

11. Chen S, Huang V, Xu X, et al. Widespread and functional RNA circularization in localized prostate cancer. Cell. 2019;176 (4):831-843. doi:10.1016/j.cell.2019.01.025

12. Hsiao KY, Lin YC, Gupta SK, et al. Noncoding effects of circular RNA CCDC66 promote colon cancer growth and metastasis. Cancer Res. 2017;77(9):2339-2350. doi:10.1158/0008-5472.CAN-16-1883

13. Yu J, Xu QG, Wang ZG, et al. Circular RNA cSMARCA5 inhibits growth and metastasis in hepatocellular carcinoma. $J$ Hepatol. 2018;68(6):1214-1227. doi:10.1016/j.jhep.2018.01.012

14. Han J, Meng J, Chen S, et al. YY1 complex promotes quaking expression via super-enhancer binding during EMT of hepatocellular carcinoma. Cancer Res. 2019;79(7):1451-1464. doi:10.1158/00085472.CAN-18-2238

15. Wang R, Zhang S, Chen X, et al. CircNT5E acts as a sponge of miR-422a to promote glioblastoma tumorigenesis. Cancer Res. 2018;78(17):4812-4825. doi:10.1158/0008-5472.CAN-18-0532

16. Xu Z, Tie X, Li N, Yi Z, Shen F, Zhang Y. Circular RNA hsa_circ_0000654 promotes esophageal squamous cell carcinoma progression by regulating the miR-149-5p/IL-6/STAT3 pathway. IUBMB Life. 2019.

17. Song $\mathrm{H}, \mathrm{Xu} \mathrm{D}$, Shi $\mathrm{P}$, et al. Upregulated circ RNA hsa circ_0000337 promotes cell proliferation, migration, and invasion of esophageal squamous cell carcinoma. Cancer Manag Res. 2019;11:1997-2006.

18. Huang H, Wei L, Qin T, Yang N, Li Z, Xu Z. Circular RNA ciRS-7 triggers the migration and invasion of esophageal squamous cell carcinoma via miR-7/KLF4 and NF-kappaB signals. Cancer Biol Ther. 2019;20(1):73-80. doi:10.1080/15384047.2018.1507254

19. Song J, Lu Y, Sun W, Han M, Zhang Y, Zhang J. Changing expression profiles of IncRNAs, circRNAs and mRNAs in esophageal squamous carcinoma. Oncol Lett. 2019;18(5):5363-5373. doi:10.3892/ol.2019.10880

20. Li Z, Yuan B, Pei Z, et al. Circ_0136474 and MMP-13 suppressed cell proliferation by competitive binding to miR-127-5p in osteoarthritis. J Cell Mol Med. 2019;23(10):6554-6564. doi:10.1111/jcmm.14400

21. Zhou ZB, Huang GX, Fu Q, et al. circRNA.33186 contributes to the pathogenesis of osteoarthritis by sponging miR-127-5p. Mol Ther. 2019;27(3):531-541. doi:10.1016/j.ymthe.2019.01.006

22. Kuang MJ, Xing F, Wang D, Sun L, Ma JX, Ma XL. CircUSP45 inhibited osteogenesis in glucocorticoid-induced osteonecrosis of femoral head by sponging miR-127-5p through PTEN/AKT signal pathway: experimental studies. Biochem Biophys Res Commun. 2019;509(1):255-261. doi:10.1016/j.bbrc.2018.12.116

23. Jakob F, Tony HP, Schneider D, Thole HH. Immunological detection of the oestradiol receptor protein in cell lines derived from the lymphatic system and the haematopoietic system: variability of specific hormone binding in vitro. J Endocrinol. 1992;134(3):397-404. doi:10.1677/joe.0.1340397

24. Liang J, Xu L, Zhou F, et al. MALAT1/miR-127-5p regulates osteopontin (OPN)-mediated proliferation of human chondrocytes through PI3K/Akt pathway. $J$ Cell Biochem. 2018;119(1):431-439. doi:10.1002/jcb.26200

25. Pronina IV, Loginov VI, Burdennyy AM, et al. DNA methylation contributes to deregulation of 12 cancer-associated microRNAs and 
breast cancer progression. Gene. 2017;604:1-8. doi:10.1016/j. gene.2016.12.018

26. Huan L, Bao C, Chen D, et al. MicroRNA-127-5p targets the biliverdin reductase $\mathrm{B} /$ nuclear factor-kappaB pathway to suppress cell growth in hepatocellular carcinoma cells. Cancer Sci. 2016;107 (3):258-266. doi:10.1111/cas.12869

27. Borlado LR, Mendez J. CDC6: from DNA replication to cell cycle checkpoints and oncogenesis. Carcinogenesis. 2008;29(2):237-243. doi:10.1093/carcin/bgm268

28. Murphy N, Ring M, Heffron CC, et al. Quantitation of CDC6 and MCM5 mRNA in cervical intraepithelial neoplasia and invasive squamous cell carcinoma of the cervix. Mod Pathol. 2005;18 (6):844-849. doi:10.1038/modpathol.3800361

29. Karakaidos P, Taraviras S, Vassiliou LV, et al. Overexpression of the replication licensing regulators $\mathrm{hCdt} 1$ and $\mathrm{hCdc} 6$ characterizes a subset of non-small-cell lung carcinomas: synergistic effect with mutant p53 on tumor growth and chromosomal instability-evidence of E2F-1 transcriptional control over hCdt1. Am J Pathol. 2004;165 (4):1351-1365. doi:10.1016/S0002-9440(10)63393-7

30. Feng CJ, Li HJ, Li JN, Lu YJ, Liao GQ. Expression of Mcm7 and Cdc6 in oral squamous cell carcinoma and precancerous lesions. Anticancer Res. 2008;28(6A):3763-3769.

\section{Publish your work in this journal}

OncoTargets and Therapy is an international, peer-reviewed, open access journal focusing on the pathological basis of all cancers, potential targets for therapy and treatment protocols employed to improve the management of cancer patients. The journal also focuses on the impact of management programs and new therapeutic

Submit your manuscript here: https://www.dovepress.com/oncotargets-and-therapy-journal agents and protocols on patient perspectives such as quality of life, adherence and satisfaction. The manuscript management system is completely online and includes a very quick and fair peer-review system, which is all easy to use. Visit http://www.dovepress.com/ testimonials.php to read real quotes from published authors. 